\title{
Association of CSMDI with Tumor Mutation Burden and Other Clinical Outcomes in Gastric
} Cancer

\author{
Xuning Wang $\mathbb{D}^{1,2, *}$ \\ Shixiang Wang ${ }^{3, *}$ \\ Yalin Han ${ }^{1,4}$ \\ Maolin $\mathrm{Xu}^{\mathrm{I}}$ \\ Peng Li ${ }^{\prime}$ \\ Mu Ke ${ }^{\prime}{ }^{\prime}$ \\ Zhipeng Teng' \\ Pu Huang' \\ Ziyan Diao ${ }^{2,5}$ \\ Yongfeng Yan' \\ Qingyu Meng' \\ Yanshen Kuang' \\ Wei Zheng' \\ Hongyi Liu' \\ Xuesong $\mathrm{Liu}^{3}$ \\ Baoqing Jia'
}

'The Air Force Hospital of Northern Theater PLA, Shenyang, People's Republic of China; ${ }^{2}$ Department of General Surgery, The First Center of Chinese PLA General Hospital, Beijing, People's Republic of China; ${ }^{3}$ School of Life Science and Technology, ShanghaiTech University, Shanghai, People's Republic of China; ${ }^{4}$ PLA Rocket Force Characteristic Medical Center, Beijing, People's Republic of China; ${ }^{5}$ Chinese PLA Medical School, Beijing,

People's Republic of China

*These authors contributed equally to this work

Correspondence: Baoqing Jia

Department of General Surgery, The First

Center of Chinese PLA General Hospital,

Beijing, People's Republic of China

Email baoqingii@।26.com

Xuesong Liu

School of Life Science and Technology,

ShanghaiTech University, Shanghai, People's

Republic of China

Email liuxs@shanghaitech.edu.cn
Background: Immunotherapy is considered as a powerful and promising clinical approach for the treatment of gastric cancer (GC). However, it is still challenging to precisely screen patients who potentially benefit from immune checkpoint therapy (ICT). Identification of potential biomarkers for selecting patients sensitive to immunotherapy was urgently needed. Methods: Public sequence data and corresponding clinical data were used to explore the potential biomarkers for immunotherapy.

Results: We found that CSMD1 is the most frequently mutated gene and its mutation is highly correlated with prognosis in gastric cancer patients. Interestingly, patients with mutated CSMD1 exhibit a high mutation burden and upregulated PDL1 expression. The ratio of microsatellite instability (MSI) in the CSMD1 mutation cohort was higher than that in the cohort without CSMD1 mutation. Furthermore, patients with CSMD1 mutation have been found to possess a higher number of activated CD4+ T cells and neoantigens.

Conclusion: CSMD1 mutation may act as a novel biomarker for assessing the survival and immune therapy response in patients with gastric cancer.

Keywords: CSMD1, PDL1, tumor mutation burden, gastric cancer

\section{Introduction}

Gastric cancer (GC) is the fifth most prevalent cancer and the third leading cause of cancer-related deaths worldwide. Despite the advances in cancer treatment, the survival rates of GC patients have not improved significantly, as the 5-year survival rate is reported to be less than $30 \%$ worldwide. ${ }^{1}$ Immunotherapy offers a powerful and promising clinical approach for the treatment of GC patients. However, different clinical responses from different patients and the risk of side effects of immune-based therapies are still unclear. Moreover, the causes underlying different outcomes in patients with similar pathological and physiological characteristics need further research. Therefore, there is an urgent need to identify new biomarkers and therapeutic targets to predict the potential response of immune checkpoint therapy (ICT). Here, we report a gene, CSMD1, which may act as a potential biomarker for assessing the immune therapy response.

CSMD1 is a member of the CSMD gene family, which has two other structurally similar proteins, including CSMD2 and CSMD $3 .{ }^{2-4}$ CSMD1 is involved in the classical complement pathway. ${ }^{5,6}$ A large population study has shown that the variants of CSMD1 (SNP: rs10503253) are associated with cognitive function in Europeans. ${ }^{7}$ However, the function of this particular variant of CSMD1 is still controversial in other populations. ${ }^{8,9}$ CSMD1 gene variants have also been 
associated with Parkinson's disease. ${ }^{10}$ These results indicated the involvement of CSMD1 in nerve system disease. Moreover, research on CSMD1 mainly focuses on carcinoma. CSMD1 gene is located in the short arm of chromosome 8 (8p23) and is frequently found to be downregulated in several cancers. Knocking down CSMD1 increased the proliferation, cell migration, and invasiveness of breast cancer cell lines. ${ }^{11}$ A study revealed that the CSMD family genes were downregulated in colon cancer. ${ }^{12}$ The expression of CSMD1 and CSMD2 was also associated with overall survival. Furthermore, the expression of CSMD2 and CSMD3 was associated with differentiation and lymphatic invasion. CSMD1 alterations were associated with earlier clinical presentation in colon cancer, which implied that CSMD1 is a tumor suppressor gene. ${ }^{13}$ Loss of CSMD1 was also found in several other cancer types, such as head and neck squamous cell carcinoma (50\%), lung (46\%) and breast (55\%) cancer, lung squamous cell carcinoma (29\%) and lung basal cell carcinoma (17\%), which has been evaluated using array-based comparative genomic hybridization. ${ }^{14}$ In melanoma, CSMD1 exhibited antitumor activity both in vivo and in vitro, induced phosphorylation (p-Smad3), and activated the Smad pathway. ${ }^{15}$ In summary, these studies indicate that CSMD1 is associated with the immune and nervous systems. Loss of CSMD1 frequently occurs in several cancers; thus, it might be a potential tumor suppressor gene.

In this study, we systematically analyzed the exon sequence data of GC patients and found an association of CSMD1 with immune therapy biomarkers.

\section{Materials and Methods}

\section{Data Collection and Processing}

The somatic mutation data was downloaded from The Cancer Genome Atlas (TCGA ${ }^{\circ}$ data portal (https://portal. gdc.cancer.gov/). TCGA provided genome mutation data analyzed using four different software packages. Here, we selected the file analyzed using mutect2, parts of the gatk workflow (https://software.broadinstitute.org/gatk/). In this study, we included all the small variants present in the downloaded somatic mutation file. ICGC (International Cancer Genome Consortium) mutation data, which we excluded from the TCGA data, were analyzed through the website (https://icgc.org/). The gene expression data (FPKM, Fragments Per Kilobase Million) and clinical data were downloaded from Xena (https://xena.ucsc.edu/). The neoantigen data of stomach cancer in TCGA were downloaded from TCIA (https://tcia.at/). All the data used in this study were obtained from public databases, and thus, it was not required to obtain additional informed consent. All the data were downloaded from a public database that has was allowed to be used in other studies (https://www. cancer.gov/about-nci/organization/ccg/research/structuralgenomics/tcga/using-tcga/citing-tcga). Thus, the research was exempt from approval by the local ethics review board.

\section{Association of CSMDI Mutation and Clinical Factors}

We used GenVisR, ${ }^{16}$ an $\mathrm{R}$ package, to analyze the mutation data and calculated the tumor mutation burden for each patient. All patients were classified into the CSMD1 mutation group or the no-mutation group based on the status of CSMD1. Tumor mutation burden (TMB) was compared between the two groups using the Wilcoxon rank sum test. The expression of PDL1 was compared using the Student's $t$-test. Meanwhile, the chi-square test was used to compare the ratio of high microsatellite instability (MSI-H). Kaplan-Meier analysis was used to analyze the survival of the two groups. The TMB correlative factors were analyzed using a general linear model and the survival-related factors by multivariate Cox proportional regression. All data were analyzed using $\mathrm{R}$.

\section{Association of CSMDI Mutation with Tumor Immune Microenvironment}

The TIMER database was used to analyze the infiltration of immune cells based on the transcriptomics data. Neoantigens were calculated using the R package, neopeptides. We compared the difference in the number of neoantigens and dissimilarity in mutated CSMD1 (MC) and wild-type CSMD1 (WC).

\section{Results}

\section{Mutation Pattern of CSMDI}

We analyzed the overall mutation pattern in GC and listed the top 17 mutated genes (Figure 1A). CSMD1 was one of the most frequently mutated genes ranked 13th in GC (Figure 1A). ICGC data also showed a similar mutation pattern in GC (Supplementary Figure S1). Among the stomach cancer samples with CSMD1 mutations, the genes related to the maintenance of genomic integrity, DNA replication proofreading, and mismatch repair, such 


\section{A}
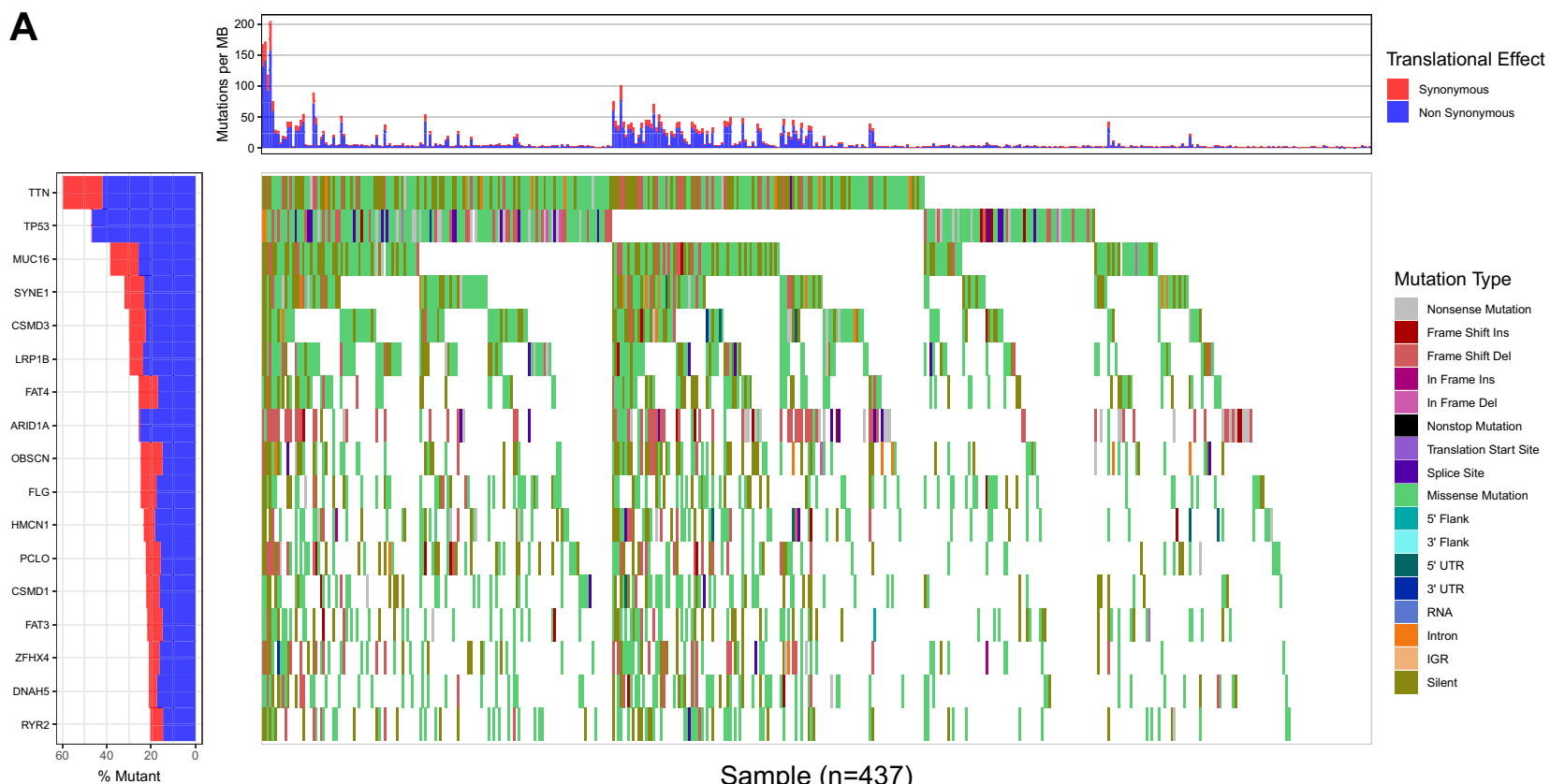

(1)

Sample $(n=437)$

B
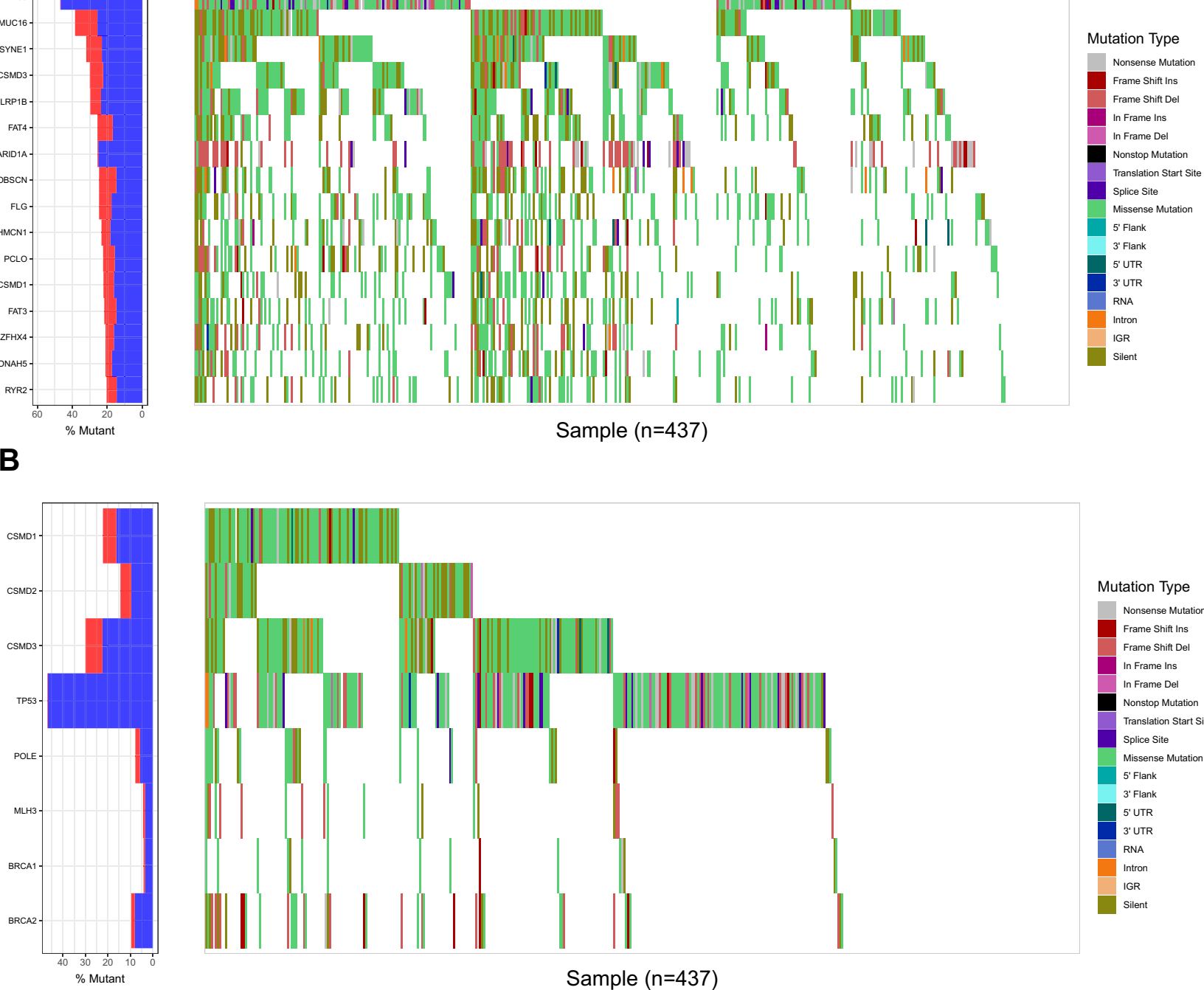

Figure I The mutation pattern of CSMDI in gastric cancer. (A) CSMDI was I3th mutated gene in gastric cancer. (B) Mutation pattern of CSMD gene family and genes associated with genomic instability TCGA.

as POLE, MLH3, and BRCA1/2 were also found to be mutated (Figure 1B).

\section{Association of CSMDI Mutation with TMB, and PDLI Expression and Survival}

The main response indicators to immune checkpoint inhibitors involved TMB, deficient mismatch repair (dMMR), and high expression of PDL1. We examined whether CSMD1 mutation correlated with these factors. We initially divided patients into two groups based on CSMD1 mutation status as follows: mutated CSMD1 (MC) and wild-type CSMD1 (WC). Interestingly, we found TMB in CSMD1 mutated group was higher than that in the wildtype group (Figure 2A). The average TMB of MC and WC was 29.91 (95\% confidence interval [CI], 21.60, 38.22) and 7.96 (95\% CI, 6.53, 9.40), (Wilcoxon test, $\mathrm{P}<2.2 \mathrm{e}-$ 16), respectively (Figure 2A). We also compared the expression of PDL1 between the two groups: MC $1.56 \pm$ 1.25 , WC $1.23 \pm 0.73$ (Figure 2B). The ratio of MSI-H in MC (40\%) was much higher than that in the WC group (12\%) (Figure $2 \mathrm{C}, \chi^{2}$ test, $\mathrm{P}=1.293 \mathrm{e}-07$ ). Furthermore, patients with $\mathrm{MC}$ had a better survival outcome than $\mathrm{WC}$ 

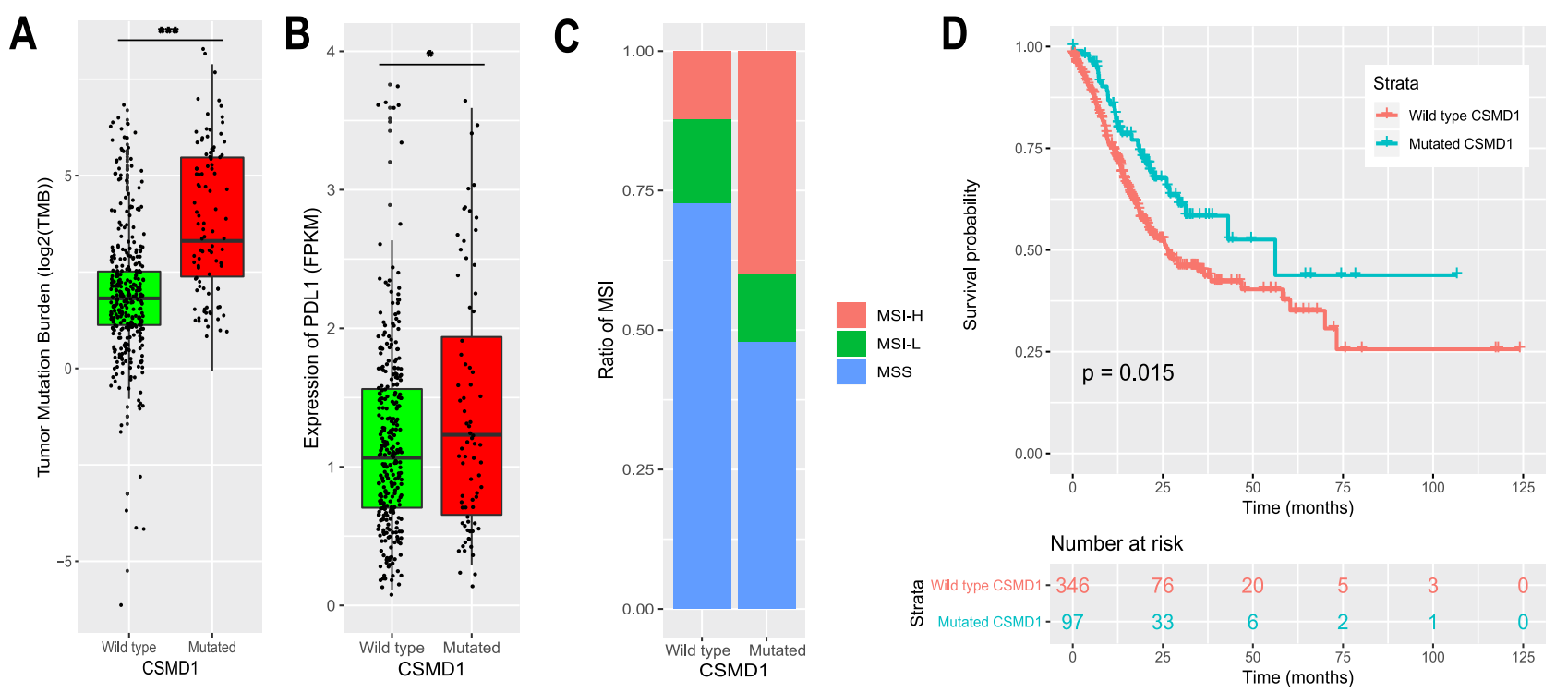

Figure 2 Association of CSMDI mutation with TMB, expression of PDLI, MSI and survival. (A) TMB of gastric cancer classified by CSMDI mutation status. (B) Expression of PDLI with CSMDI mutation was upregulated. (C) The ratio of MSI-H in CSMDI mutation group was increased in GC (D) patients with CSMDI mutation had better survival than patients without CSMDI mutation. (*p<0.05, *** $\mathrm{p}<0.00 \mathrm{I})$.

(Figure 2D; median overall survival, $56.2[95 \% \mathrm{CI}$, 31.3-NA] vs 26.4 [95\% CI, 21.1-46.9] months; Log rank test, $\mathrm{P}=0.0015$ ).

\section{Association of CSMDI Mutation with TMB and Survival Outcome}

We treated CSMD1 mutation status as an independent factor. A general linear model was applied to analyze the relationship between TMB and other clinical factors, including CSMD1 mutation status. The results are shown in Figure 3A. Here, TMB was associated with age $(\mathrm{P}=$ 0.003), number of lymph nodes ( $\mathrm{P}<0.003)$, and CSMD1 status $(\mathrm{P}<0.001)$ in this study. It has been shown in Figure $3 \mathrm{~B}$ that age and the number of lymph and CSMD1 mutation status (hazard ratio: $0.63,95 \% \mathrm{CI}$ : 0.41. $0.96, \mathrm{P}=0.03$ ) were correlated with survival.

\section{Association of CSDMI Mutation with Tumor Immune Microenvironment}

We downloaded the immune infiltration status of GC patients from the TIMER database and compared the differences between the two groups (MC vs WC). The cohort in $\mathrm{MC}$ exhibited higher infiltration of activated $\mathrm{CD}^{+}$ $\mathrm{T}$ cells (Figure 4A). However, other immune cells were not statistically significant. Furthermore, we analyzed neoantigens in GC patients. We found that the number of neoantigen peptides in $\mathrm{MC}$ was significantly higher than that in WC (Figure 4B). The peptide of IEDB in MC was also significantly higher than that in WC (Figure 4C), and neoantigen dissimilarity showed a similar trend (Figure 4D).

\section{Discussion}

Recent reports have demonstrated that the concept of tumor mutation burden in cancers highlighted the fact that mutations in specific factors are associated with the development and progression of cancer, serving as important biomarkers for assessing the survival, immunotherapy response, and even providing a set of valuable targets for cancer therapy. Several trials have shown a correlation between patients with high TMB and better responses to immunotherapy agents. ${ }^{17,18}$ According to previous studies, the main biomarkers for immune checkpoint inhibitor therapy response include $\mathrm{TMB},{ }^{19,20}$ expression of PDL1, ${ }^{21-23} \mathrm{dMMR},{ }^{24,25}$ and CD8+ T-cell infiltration. ${ }^{26}$ Thus, genes associated with $\mathrm{TMB}$, the expression of PDL1, dMMR, and/or CD8+ $\mathrm{T}$ cell infiltration may act as potential biomarkers for assessing immune checkpoint inhibitor therapy response.

CSDM1 plays a crucial role in several cancers, such as head and neck carcinomas and colon cancers. Some studies have reported that CSMD1 can be considered as a tumor suppressor gene.

In our study, we analyzed TCGA cohort and found that CSMD1 was one of frequently mutated genes in GC, and 

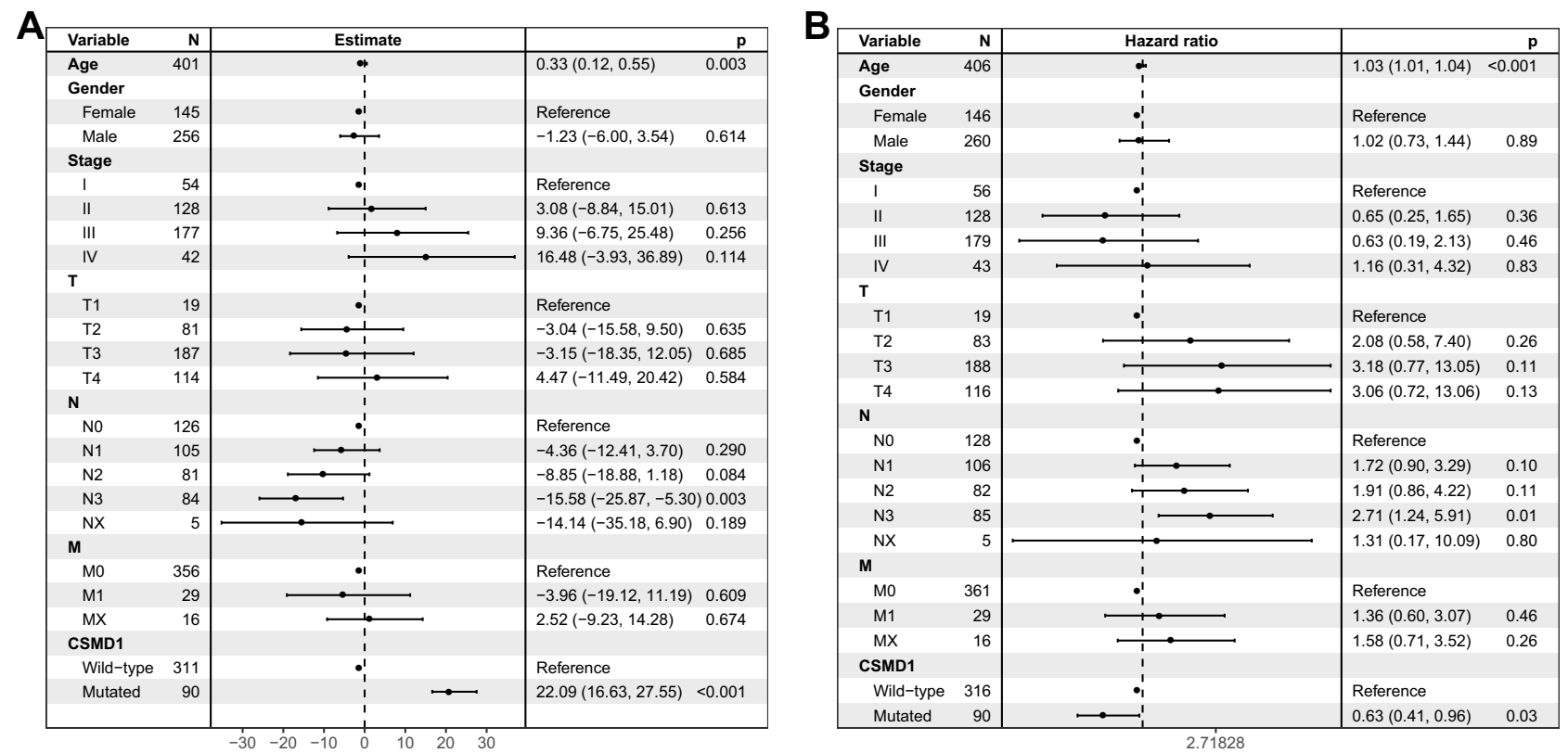

Figure 3 Correlation of CSMDI mutation and TBM and survival outcome. (A) TMB was related to CSMDI mutation according to general linear analysis. (B) CSMDI mutation was associated with survival outcome through multivariate Cox regression analysis.
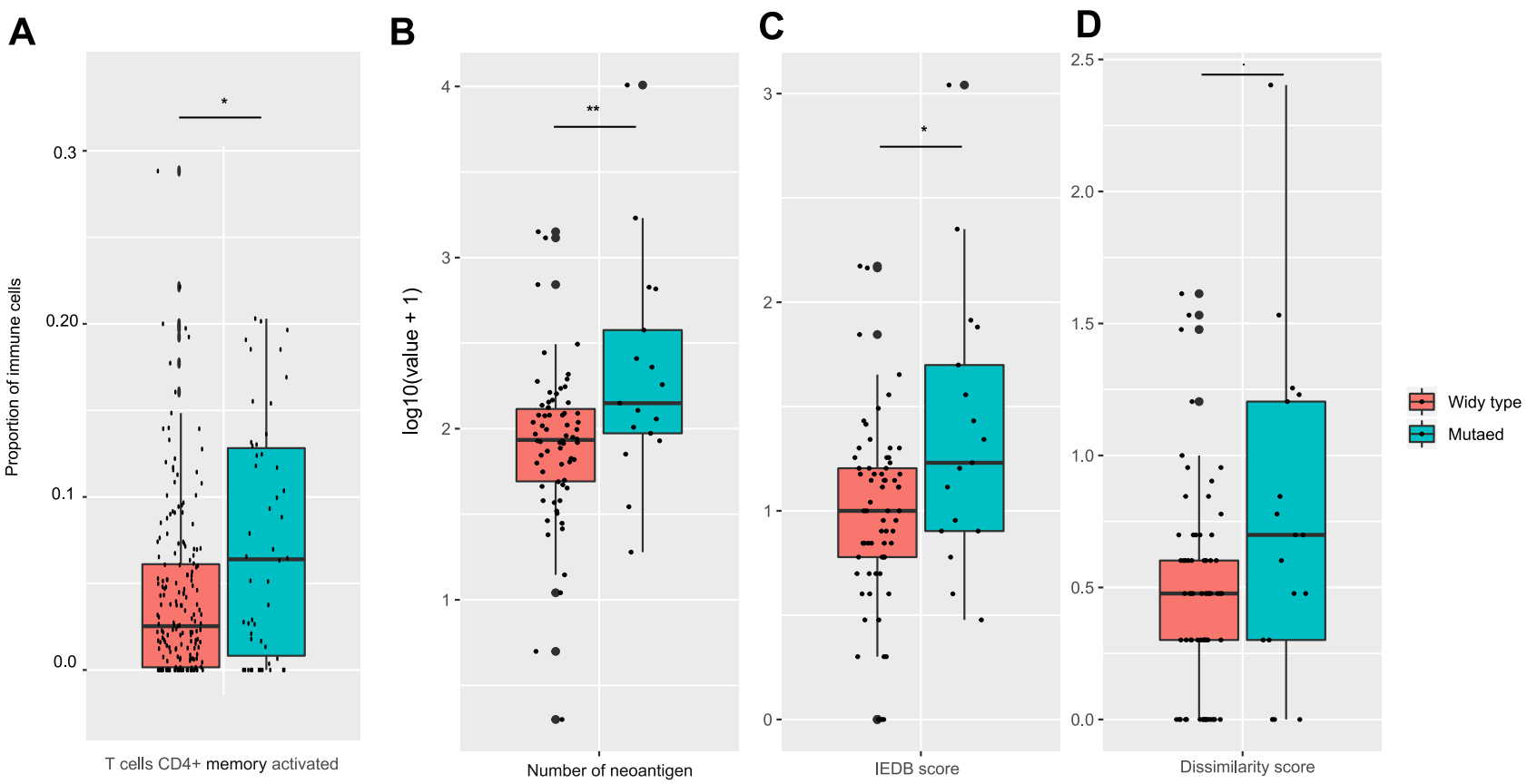

Figure 4 CSMDI mutation was associated with neoantigens. Compared with non-CSMDI mutation patients, the CSMDI mutation group has more activated CD4+ T cells (A), more neoantigens (B and $\mathbf{C}$ ) and dissimilarity (D). $\left({ }^{*} p<0.05,{ }^{* *} p<0.0\right.$ I).

patients with CSMD1 mutations often harbored other gene mutations, which played a crucial role in the maintenance of genomic integrity. In the ICGC dataset, we obtained similar results, which further indicated that CSMD1 may be an important gene in GC. CSMD1 mutation was not only associated with higher TMB but also with higher expression of PDL1 and MSI-H. CSMD1 may act as a potential biomarker for assessing immune therapy response in patients with GC. It might serve as a biomarker of anti-PDL1 drug response in clinical 
practice. Therefore, immune checkpoint blockade-related drugs may be beneficial to patients with CSMD1 mutations. Furthermore, patients with the CSMD1 mutation exhibit a better survival than those without the CSMD1 mutation, implying that the CSDM1 mutation could be used as a potential biomarker of overall survival. The multivariate Cox regression model also indicated that CSMD1 is associated with overall survival.

We further analyzed the relationship between CSMD1 mutation and TMB through a general linear model, which indicated the association of CSMD1 with TMB.

These results further implied that the CSMD1 mutation serves as a biomarker for assessing the response of immune checkpoint blockade-related drug. The potential ability of CSDM1 in GC as an immune block inhibitor should be taken into consideration. In this study, two limitations were noted. First, somatic mutation data were derived from a single dataset and are not validated in other independent datasets. Second, we did not investigate the mechanisms underlying these results, and further experiments are required to be performed to confirm them further.

\section{Conclusion}

CSMD1 mutation is associated with TMB, dMMR, and the expression of PDL1. Patients with CSMD1 mutation exhibit better survival and possess more neoantigens than patients without it.

\section{Ethical Statement}

All the data used in this study were obtained from public databases, and thus, it was not required to obtain additional informed consent.

\section{Consent for Publication}

The authors of this paper have consented this study for publication.

\section{Funding}

There is no funding to report.

\section{Disclosure}

The authors declare that they have no competing interests.

\section{References}

1. Ferlay J, Soerjomataram I, Dikshit R, et al. Cancer incidence and mortality worldwide: sources, methods and major patterns in GLOBOCAN 2012. Int $J$ Cancer. 2015;136(5):E359-E386. doi:10.1002/ijc. 29210
2. Sun PC, Uppaluri R, Schmidt AP, et al. Transcript map of the $8 \mathrm{p} 23$ putative tumor suppressor region. Genomics. 2001;75(1-3):17-25. doi:10.1006/geno.2001.6587

3. Lau WL, Scholnick SB. Identification of two new members of the CSMD gene family. Genomics. 2003;82(3):412-415. doi:10.1016/ S0888-7543(03)00149-6

4. Shimizu A, Asakawa S, Sasaki T, et al. A novel giant gene CSMD3 encoding a protein with CUB and sushi multiple domains: a candidate gene for benign adult familial myoclonic epilepsy on human chromosome 8q23.3-q24.1. Biochem Biophys Res Commun. 2003;309(1):143-154. doi:10.1016/S0006-291X(03)01555-9

5. Kraus DM, Elliott GS, Chute H, et al. CSMD1 is a novel multiple domain complement-regulatory protein highly expressed in the central nervous system and epithelial tissues. J Immunol. 2006;176 (7):4419-4430. doi:10.4049/jimmunol.176.7.4419

6. Escudero-esparza A, Kalchishkova N, Kurbasic E, Jiang WG, Blom AM. The novel complement inhibitor human CUB and Sushi multiple domains 1 (CSMD1) protein promotes factor I-mediated degradation of $\mathrm{C} 4 \mathrm{~b}$ and $\mathrm{C} 3 \mathrm{~b}$ and inhibits the membrane attack complex assembly. FASEB j. 2013;27(12):5083-5093. doi:10.1096/fj.13230706

7. Athanasiu L, Giddaluru S, Fernandes C, et al. A genetic association study of CSMD1 and CSMD2 with cognitive function. Brain Behav Immun. 2017;61:209-216. doi:10.1016/j.bbi.2016.11.026

8. Liu Y, Cheng Z, Wang J, et al. No association between the rs 10503253 polymorphism in the CSMD1 gene and schizophrenia in a Han Chinese population. BMC Psychiatry. 2016;16:206. doi:10.1186/s12888-016-0923-5

9. Liu W, Liu F, Xu X, Bai Y. Replicated association between the European GWAS locus rs10503253 at CSMD1 and schizophrenia in Asian population. Neurosci Lett. 2017;647:122-128. doi:10.1016/j. neulet.2017.03.039

10. Shahmohammadibeni N, Rahimi-aliabadi S, Jamshidi J, et al. The analysis of association between SNCA, HUSEYO and CSMD1 gene variants and Parkinson's disease in Iranian population. Neurol Sci. 2016;37(5):731-736. doi:10.1007/s10072-015-2420-x

11. Zhang Y, Wang S, Kang W, et al. CREPT facilitates colorectal cancer growth through inducing Wnt/ $\beta$-catenin pathway by enhancing $\mathrm{p} 300$ mediated $\beta$-catenin acetylation. Oncogene. 2018;37(26):3485-3500. doi:10.1038/s41388-018-0161-z

12. Zhang R, Song C. Loss of CSMD1 or 2 may contribute to the poor prognosis of colorectal cancer patients. Tumour Biol. 2014;35 (5):4419-4423. doi:10.1007/s13277-013-1581-6

13. Shull AY, Clendenning ML, Ghoshal-gupta S, et al. Somatic mutations, allele loss, and DNA methylation of the Cub and Sushi Multiple Domains 1 (CSMD1) gene reveals association with early age of diagnosis in colorectal cancer patients. PLoS One. 2013;8(3): e58731. doi:10.1371/journal.pone.0058731

14. Ma C, Quesnelle KM, Sparano A, et al. CharacterizationCSMD1 in a large set of primary lung, head and neck, breast and skin cancer tissues. Cancer Biol Ther. 2014;8(10):907-916. doi:10.4161/ cbt.8.10.8132

15. Tang MR, Wang YX, Guo S, Han SY, Wang D. CSMD1 exhibits antitumor activity in A375 melanoma cells through activation of the Smad pathway. Apoptosis. 2012;17(9):927-937. doi:10.1007/s10495012-0727-0

16. Skidmore ZL, Wagner AH, Lesurf R, et al. GenVisR: genomic visualizations in R. Bioinformatics. 2016;32(19):3012-3014. doi:10.1093/bioinformatics/btw325

17. Samstein RM, Lee CH, Shoushtari AN, et al. Tumor mutational load predicts survival after immunotherapy across multiple cancer types. Nat Genet. 2019. doi:10.1038/s41588-018-0312-8

18. Topalian SL, Taube JM, Anders RA, Pardoll DM. Mechanism-driven biomarkers to guide immune checkpoint blockade in cancer therapy. Nat Rev Cancer. 2016;16(5):275. doi:10.1038/nrc.2016.36 
19. Snyder A, Makarov V, Merghoub T, et al. Genetic basis for clinical response to CTLA-4 blockade in melanoma. $N$ Engl J Med. 2014;371 (23):2189-2199. doi:10.1056/NEJMoa1406498

20. Wang VE, Urisman A, Albacker L, et al. Checkpoint inhibitor is active against large cell neuroendocrine carcinoma with high tumor mutation burden. J Immunother Cancer. 2017;5(1):75. doi:10.1186/ s40425-017-0281-y

21. Herbst RS, Soria JC, Kowanetz M, et al. Predictive correlates of response to the anti-PD-L1 antibody MPDL3280A in cancer patients. Nature. 2014;515(7528):563-567. doi:10.1038/nature14011

22. Buder-bakhaya K, Hassel JC. Biomarkers for clinical benefit of immune checkpoint inhibitor treatment-a review from the melanoma perspective and beyond. Front Immunol. 2018;9:1474. doi:10.3389/ fimmu.2018.01474
23. Shukuya T, Carbone DP. Predictive markers for the efficacy of anti-PD-1/PD-L1 antibodies in lung cancer. $J$ Thorac Oncol. 2016;11(7):976-988. doi:10.1016/j.jtho.2016.02.015

24. Le DT, Durham JN, Smith K, et al. Mismatch repair deficiency predicts response of solid tumors to PD-1 blockade. Science. 2017;357(6349):409-413. doi:10.1126/science.aan6733

25. Lee V, Murphy A, Le DT, Diaz LA JR. Mismatch repair deficiency and response to immune checkpoint blockade. Oncologist. 2016;21 (10):1200-1211.

26. Tang H, Wang Y, Chlewicki LK, et al. Facilitating T cell infiltration in tumor microenvironment overcomes resistance to PD-L1 blockade. Cancer Cell. 2016;29(3):285-296. doi:10.1016/j.ccell.2016.02.004

\section{Publish your work in this journal}

The International Journal of General Medicine is an international, peer-reviewed open-access journal that focuses on general and internal medicine, pathogenesis, epidemiology, diagnosis, monitoring and treatment protocols. The journal is characterized by the rapid reporting of reviews, original research and clinical studies across all disease areas. The manuscript management system is completely online and includes a very quick and fair peer-review system, which is all easy to use. Visit http://www.dovepress.com/ testimonials.php to read real quotes from published authors. 